\title{
Unique and Minimum Distance Decoding of Linear Codes with Reduced Complexity
}

\author{
D. Spasov (spasov.dejan@gmail.com)
}

\begin{abstract}
We show that for (systematic) linear codes the time complexity of unique decoding is $O\left(n^{2} q^{n R H(\delta / 2 / R)}\right)$ and the time complexity of minimum distance decoding is $O\left(n^{2} q^{n R H(\delta / R)}\right)$. The proposed algorithm inspects all error patterns in the information set of the received message of weight less than $d / 2$ or $d$, respectively.
\end{abstract}

Index Terms-nearest neighbor decoding, unique decoding, bounded distance decoding, minimum distance decoding.

\section{INTRODUCTION}

I ET $C$ is a systematic linear code with parameters

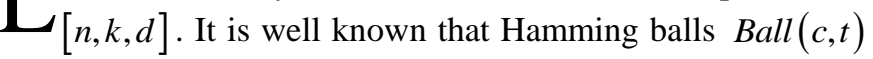
with radius $t=\lfloor(d-1) / 2\rfloor$ around the codewords $c \in C$ are disjoint. Let $y$ is the received message. Then the Unique Decoding strategy is to find the codeword $c_{y} \in C$, such that $y \in \operatorname{Ball}\left(c_{y}, t\right)$, or return incomplete decoding, i.e. $y \notin \operatorname{Ball}(c, t) \forall c \in C$. Trivial way to do this is to inspect all $q^{k}$ codewords and return $c_{y}$ such that $d\left(y, c_{y}\right) \leq t$. The time complexity of this approach is $O\left(n q^{R n}\right)$. Another alternative for unique decoding, with time complexity $O\left(n q^{H(\delta / 2) n}\right)$, is to inspect all $V(n, t)$ error patterns $e$ and find the pattern such that $y-e$ is a codeword. Minimum Distance Decoding, on the other hand, inspects all $q^{k}$ codewords and returns $c_{y} \in C$ such that $d\left(y, c_{y}\right)$ is minimal or it inspects all error patterns of weight less than the covering radius [1].

In the following section we will show that unique decoding can be done by inspecting all $V(k, t)$ error patterns in the information set of the received message $y$. Then we will generalize this algorithm to perform Minimum Distance Decoding.

\section{The AlgORITHM}

We will use $\langle a \mid b\rangle$ to denote concatenation of two vectors, such that $a$ belongs to the information set and $b$ belongs to the check set of a codeword. Let the message $x$ is encoded in the codeword $c_{x}=\langle x \mid r\rangle$ and sent over a noisy channel. Let the random error pattern is denoted with $e=\langle v \mid u\rangle$ and the received word is denoted with $y=\left\langle y_{x} \mid y_{r}\right\rangle$. The unique decoding algorithm, below, inspects all error patterns in the information set $e=\langle v \mid 0\rangle$ with weight $w t(e) \leq t$ and outputs the message $\langle x \mid u\rangle$ if $y$ belongs to some $\operatorname{Ball}(c, t)$ :

Unique_Decoding $(y)$

$$
\begin{aligned}
& \text { Let } t=\lfloor(d-1) / 2\rfloor \\
& \text { 1. find the syndrome } s_{Y}=H y^{T} \\
& \text { 2. if } w t\left(s_{y}\right) \leq t \text { return } y \\
& \text { 3. foreach vector } e=<v \mid 0>\text { s.t. } w t(e) \leq t \\
& \text { a. find } s_{e}=H e^{T} \\
& \text { b. if } w t(e)+w t\left(s_{Y}-s_{e}\right) \leq t \\
& \text { return } y^{-e} \\
& \text { 4. return }-1 / / \text { incomplete decoding }
\end{aligned}
$$

Proposition 1: The Unique_Decoding(y) algorithm can remove any error pattern of weight $\leq t$ from the received message $y$.

Proof: Let $e_{v}=\langle v \mid 0\rangle, w t\left(e_{v}\right) \leq t$, is the coset leader and $s_{v}=H e_{v}^{T}$ is the syndrome of a coset. Let assume that the pairs $\left(s_{v}, e_{v}\right)$ are explicitly known; for example, they are stored in a look-up table.

We will consider the error pattern $e=\langle v \mid u\rangle$ as a linear combination of two vectors

$$
e=\langle v \mid 0\rangle+\langle 0 \mid u\rangle=e_{v}+e_{u}
$$

Since $w t\left(e_{u}\right) \leq t$ and $s_{u}=H e_{u}^{T}=u$, we can say that $e_{u}$ is 
the leader and $u$ is the syndrome of the same coset. Hence, the syndrome $s$ of the received message $y$ is

$$
s=H y^{T}=H\left(c_{x}+e_{v}+e_{u}\right)^{T}=s_{v}+u
$$

From (2), we can formulate the decoding strategy: for each $e_{v}$ in the table $\left(s_{v}, e_{v}\right)$ denote with $x=y-e_{v}$ and compute the syndrome

$$
s_{x}=H x^{T}
$$

If

$$
w t\left(e_{v}\right)+w t\left(s_{x}\right) \leq t
$$

then the error pattern that corrupted the message is

$$
e=\left\langle e_{v} \mid s_{x}\right\rangle
$$

In worst case, the algorithm will check all $V_{q}(k, t)$ error patterns. So the time complexity is upper-bounded by

$$
O\left(n^{2} q^{R H\left(\frac{\delta}{2 R}\right) n}\right)
$$

If we use the fact that for long random linear codes the covering radius is equal to $d$, where $d$ is the largest integer solution of the Gilber-Varshamov inequality [2]. Then we can formulate Minimum Distance Decoding algorithm that inspects all error patterns of weight less than $d$ in the information set:

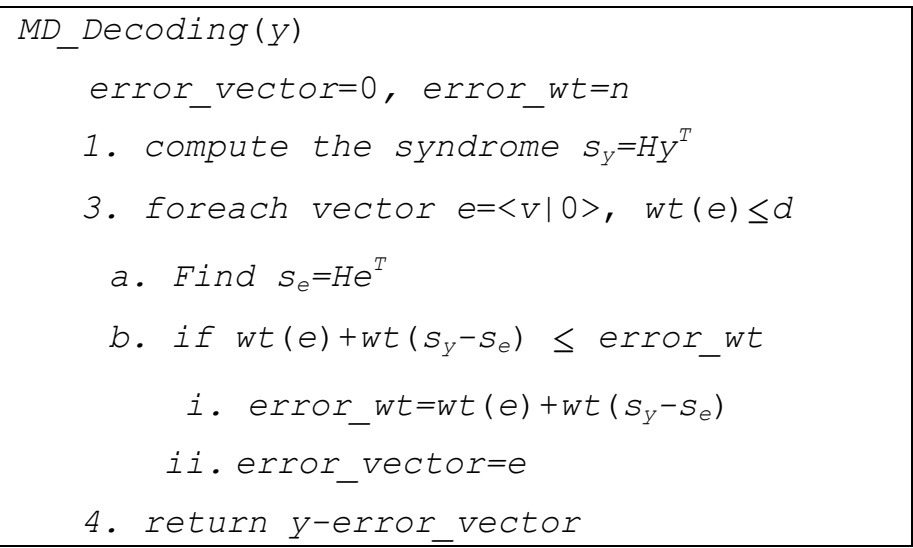

The proof of correctness of the above algorithm is similar to the proof of proposition 1. Thus the time complexity of MDD decoding is

$$
O\left(n^{2} q^{R H\left(\frac{\delta}{R}\right) n}\right)
$$

and this result improves some of the previously known bounds on MD decoding found in [1], [2], [3], or [4].

On the other hand, pairs $\left(s_{v}, e_{v}\right)$ need not to be stored in a look-up table, but they can be listed by divide-and-conquer strategy in the course of decoding. Therefore, the space complexity of Unique Decoding $(y)$ and $M D \_$Decoding $(y)$ is proportional with the dimension of the generator matrix, i.e. $O\left(n^{2}\right)$.

\section{REFERENCES}

[1] A. Barg, "Complexity issues in coding theory," Handbook of Coding Theory, vol. 1, Elsevier Science, 1998, pp. 649-754

[2] A. Barg, E. Krouk, and H. van Tilborg, "On the complexity of Minimum Distance Decoding of Long Linear Codes," IEEE Transactions on Information Theory, vol. 45, no. 5, pp. 1392-1405, July 1999.

[3] I. Dumer, "Suboptimal decoding of linear codes: Partition technique," IEEE Trans. Inform. Theory, vol. 42, no. 6, pp. 1971-1986, 1996.

[4] L. Levitin and C. R. P. Hartmann, "A new approach to the general minimum distance decoding problem: The zero-neighbors algorithm," IEEE Trans. Inform. Theory, vol. IT-31, pp. 378-384, May 1985. 